\title{
A Pareto Optimization Applied in Power Control of Wireless Sensor Networks
}

\author{
Hao Chen ${ }^{1}$, Xiufeng Zhang ${ }^{2}$ and Zhang $\mathrm{Li}^{2}$ \\ ${ }^{1}$ School of Computer and Information Engineering \\ Tianjin Chengjian University \\ Tianjin 300384, P.R.China \\ Emails: haochen11144@163.com \\ ${ }^{2}$ School of Management, Harbin Institute of Technology, \\ Harbin 150001,P.R.China zmlmdx@126.com
}

\begin{abstract}
In this paper, achievable rate region of two-user interference channel is studied. We use the Pareto-optimal method (e.g., specially in production and consumption) into the wireless sensor nodes transmit power allocation strategies in coupled channels. The result shows that when systems in optimality conditions, minim transmit power can arrive maximum channel throughout. What's more, achievable rate of each channel equals and it is only related the channel parameters at the moment.
\end{abstract}

Keywords: power allocation strategies, achievable rate, wireless sensor networks, Pareto optimization

\section{Introduction}

A. Interference in Communication Networks

The wireless sensor network uses universal spectrum reuse, i.e., all source nodes transmit at the same frequency channel to target nodes. In earlier wireless sensor networks, the radius was large and the communication link was mainly range limited, or equivalently noise-limited. Later networks used higher spatial reuse of the spectrum per geographical area, and the transition size decreased to accommodate the decreasing of nodes energy. Therefore, the source nodes density increased and the target nodes got closer to each other. This change shift communication links from being noise-limited to being interference-limited.

B .Capacity of the 2-user Interference Channel

We are interested in the scenario where multiple wireless links operate at the same carrier frequency and mutually interfere with one another. Specifically, we consider two transmitter-receiver pairs TX1 $\rightarrow \mathrm{RX} 1$ and TX2 $\rightarrow \mathrm{RX} 2$ that are located in the proximity of one another, so that RX1 receives significant interference from TX2 and vice versa. We focus on the transmit strategies for TX1 and TX2 that are optimum in the Pareto sense. That is, we look for pairs of transmit strategies such that if any of the strategies is changed so that the transmission rate for the link TX1 $\rightarrow \mathrm{RX} 1$ increases, then the rate for the other link TX2 $\rightarrow$ RX2 must necessarily decrease, and vice versa. If the interference is sufficiently weak, then it is optimal from a capacity point of view to treat it as additive noise [1]. Hence, Pareto-optimal transmit strategies directly correspond to Pareto-optimal signal-to-interference-and-noise ratio (SINR) pairs. 


\section{Contributions}

Prior references [2-8] have studied many questions about Pareto as assumption that the transmitting nodes and receiving nodes have use amount of complicate techniques, power control cross correlation of pseudo-random codes, etc. However, this comes at the expense of sharing messages between the transmitting nodes or between the receiving nodes, and sometimes this might not be a feasible or an efficient strategy. With design dictums of low power consumption and low complexity receivers, no complex successive decoding nor maximum likelihood decoding would be advised[9-12]. This need motivated us to propose an optimization problem about throughout of channel as following assumption. The assumption is that there are no signal cooperation between transmitters (no joint encoding)[13-15], no signal cooperation between receivers (no joint decoding), single input single output communication (SISO), and a frequency flat channel.

\section{System Model}

The 2-user interference channel is presented in Figure 1 with 2 transmitters and 2 receivers.

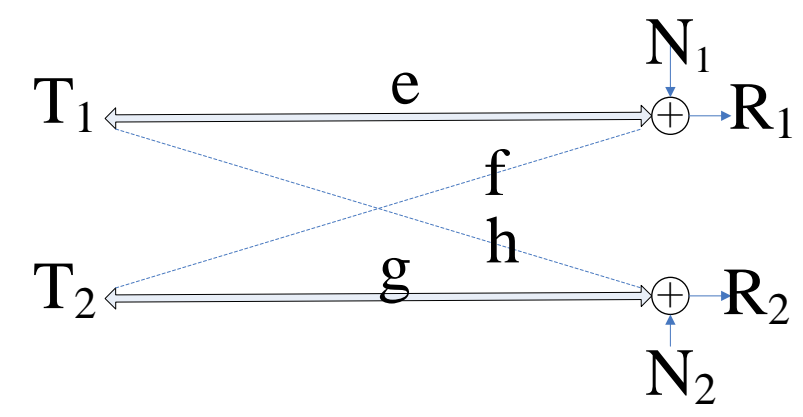

Figure1. 2-user Interference Channel Model

The $i^{\text {th }}$ transmitter transmits its signal $T_{i}$ to the intended $i^{\text {th }}$ receiver with a power $P_{i}$. The receivers have independent additive complex white Gaussian noise with a power noise variance of $\sigma_{n}^{2}$. Each transmitter is assumed to have a maximum power constraint of $P_{\max } . G_{i, j}$ denotes the channel power gain received at the $i^{\text {th }}$ receiver from the $j^{\text {th }}$ transmitter, and there are no constraints over the values or distributions of $G_{i, j}$. Therefore $G_{i, i}$ is the channel gain of the $i^{\text {th }}$ desired signal, where as $G_{i, j}, j \neq i$ represent the interfering channel gains. $M_{i}$ denotes the maximum reliable rate of communication between the $i^{\text {th }}$ transmitter and the $i^{\text {th }}$ receiver.

From the view of 2-user physical sense, transmitter-receiver function can be expressed as follows:

$$
\begin{aligned}
& M_{1}\left(P_{1}, P_{2}\right)=\log _{2}\left(1+\frac{k P_{1}}{1+l P_{2}}\right) \\
& M_{2}\left(P_{1}, P_{2}\right)=\log _{2}\left(1+\frac{s P_{2}}{1+r P_{1}}\right)
\end{aligned}
$$

The channel power gains are normalized by the noise variance and $k, l, r$ and $s$ respectively denote as follows : 


$$
k=\frac{g_{1,1}}{\sigma_{n}^{2}} \quad l=\frac{g_{1,2}}{\sigma_{n}^{2}} \quad r=\frac{g_{2,1}}{\sigma_{n}^{2}} \quad s=\frac{g_{2,2}}{\sigma_{n}^{2}}
$$

In order to further discussion and use Pareto method, the two variables $P_{1}$ and $P_{2}$ are expended four variables $P_{1,1}, P_{1,2}, P_{2,1}$ and $P_{2,2}$. The channel power gain and the noise variance will be reset and redefined a symbol, respectively.

$P_{1}=e P_{1,1}+h P_{2,1} \quad e k+h r=1$

$P_{2}=g P_{2,2}+f P_{1,2} \quad g s+f l=1$

Therefore, $M_{1}$ and $M_{2}$ can be rewritten as follows:

$$
\begin{aligned}
& M_{1}\left(P_{1,1}, P_{1,2}\right)=\log _{2}\left(1+\frac{P_{1,1}}{1+P_{1,2}}\right) \\
& M_{2}\left(P_{2,2}, P_{2,1}\right)=\log _{2}\left(1+\frac{P_{2,2}}{1+P_{2,1}}\right)
\end{aligned}
$$

Next, from the view of microeconomics, the Pareto optimal thought way is applied to analyze the relation of achievable rate and interference channel parameters.

Definition.1[4]. A rate pair $\left(M_{1}^{*}, M_{2}^{*}\right) \in R$ is Pareto optimal if there is no other rate $\operatorname{pair}\left(M_{1}, M_{2}\right) \in R$ with $\left(M_{1}, M_{2}\right) \geq\left(M_{1}^{*}, M_{2}^{*}\right)$.(The inequality is component-wise.

For notational brevity, power variable symbol can be redefined as:

$P_{1,1}=u, P_{2,1}=v, P_{2,2}=w, P_{1,2}=x$

By inserting Eq.(7) into Eq.,(3),(4), (5) and (6) we derive the following equations.

$M_{1}(s, t)=\log _{2}\left(1+\frac{u}{1+v}\right)$

$M_{2}(x, y)=\log _{2}\left(1+\frac{w}{1+x}\right)$

$P_{1}=e u+h x$

$P_{2}=g w+f v$

In mathematical optimization, the method of Lagrange multipliers provides a strategy for finding the maxima of a function subject to constraints.

Maximize Eq.(8)

Subject to Eq. (9),(10) and(11)

Lagrange multiplier $\lambda, \sigma$ and $\mu$ are introduced and the Lagrange function is constructed as Eq.(12).

$Z=M_{1}(u, v)+\lambda\left[\bar{M}_{2}-M_{2}(w, x)\right]+\sigma\left(P_{1}-e u-h x\right)+\mu\left(P_{2}-g w-f v\right)$

The objective function $Z$ differentiates with respect to $s, t, x$ and $y$, respectively. 


$$
\begin{aligned}
& \frac{\partial Z}{\partial u}=\frac{\partial M_{1}}{\partial u}-\sigma e=0 \rightarrow \frac{\partial M_{1}}{\partial u}=\sigma e \\
& \frac{\partial Z}{\partial t}=\frac{\partial C_{1}}{\partial t}-\mu b=0 \rightarrow \frac{\partial C_{1}}{\partial t}=\mu b \\
& \frac{\partial Z}{\partial w}=-\lambda \frac{\partial M_{2}}{\partial w}-\mu g=0 \rightarrow \frac{\partial M_{2}}{\partial w}=-\frac{\mu g}{\lambda} \\
& \frac{\partial Z}{\partial}=-\lambda \frac{\partial C_{2}}{\partial y}-\sigma d=0 \rightarrow \frac{\partial C_{2}}{\partial y}=-\frac{\sigma d}{\lambda}
\end{aligned}
$$

In economics, a relation is known as the fact that the Marginal Rate of Technical Substitution (MRTS) at a Pareto optimal resource allocation is the same for all consumers (here transmitter-receiver pairs).Along an isoquant, the MRTS shows the rate at which one input (e.g., transmit power of different channel) may be substituted for another, while maintaining the same level of output (e.g., achievable rate). The MRTS can also be seen as the slope of an isoquant at the point in question.

$$
\begin{aligned}
& \operatorname{MRTS}_{P_{1} P_{2}}^{M 1_{1}}=\frac{\partial M_{1}}{\partial u} / \frac{\partial M_{1}}{\partial v}=\frac{\sigma e}{\mu f} \\
& M R T S_{P_{1} P_{2}}^{M_{2}}=\frac{\partial M_{2}}{\partial w} / \frac{\partial M_{2}}{\partial x}=\frac{\mu g}{\sigma h}
\end{aligned}
$$

Reviewing the Eq.(9), $M_{2}(x, y)$ differentiates with respect to $w$ and $x$, respectively.

$$
\begin{aligned}
\frac{\partial M_{2}}{\partial w} & =\frac{1}{\ln 2 \cdot(1+w+x)} \\
\frac{\partial M_{2}}{\partial x} & =\frac{(-w)}{\ln 2 \cdot(1+w+x)(1+x)}
\end{aligned}
$$

By inserting Eq.(19) and Eq.(20) into Eq.(18), Eq.(21) is derived as follows:

$$
\frac{\mu g}{\sigma h}=-\frac{1+x}{w} \Rightarrow \frac{\mu}{\sigma}=\left(\frac{h}{g}\right) \cdot\left(-\frac{1+x}{w}\right)
$$

By Eq. (19),(20)and (21), Eq.(17)and (18) can further simplify as follows:

$$
\begin{aligned}
& \operatorname{MRTS} S_{P_{1} P_{2}}^{M_{1}}=\frac{\partial M_{1}}{\partial u} / \frac{\partial M_{1}}{\partial v}=\frac{e g}{f h} \cdot\left(\frac{-w}{1+x}\right) \\
& M R T S_{P_{1} P_{2}}^{M_{2}}=\frac{\partial M_{2}}{\partial w} / \frac{\partial M_{2}}{\partial x}=\frac{1+x}{-w} \\
& \operatorname{MRTS}_{P_{1} P_{2}}^{M_{1}}=\operatorname{MRTS} S_{P_{1} P_{2}}^{M_{2}}
\end{aligned}
$$

Eq.(24)is the Pareto optimal condition. It is the different Pareto point (here achievable rate) with respect to different transmit power. The conclusion suggests that the tangent is the minimum cost line. That is, minimum power can arrive maximum channel throughout.

From Eq.(24), Eq.(25) can be derived. 
$\frac{1+x}{-w}=\frac{e g}{f h} \cdot\left(\frac{-w}{1+x}\right) \Rightarrow \frac{w}{1+x}=\sqrt{\frac{f h}{e g}}$

By Eq.(25) inserting Eq.(9), Eq.(26) can be derived.

$\sqrt{\frac{f h}{e f}}=2^{M_{2}}-1$

Based on the same principle, we treat Eq.(9) as objective function and treat Eq.(8),(10)and(11)as constraint conditions. Similar conclusion can be derived as follows:

$\sqrt{\frac{f h}{e g}}=2^{M_{1}}-1$

We can draw a perfect conclusion from Eq.(26)and(27)

\section{Experimental Results and Analysis}

Power control (PC) mechanism have been elaborated as follows. The model for power control uses the Carrier to Interferer (C/I) ratio at the receiver as well as the receiving information power level as shown in Figure 2.

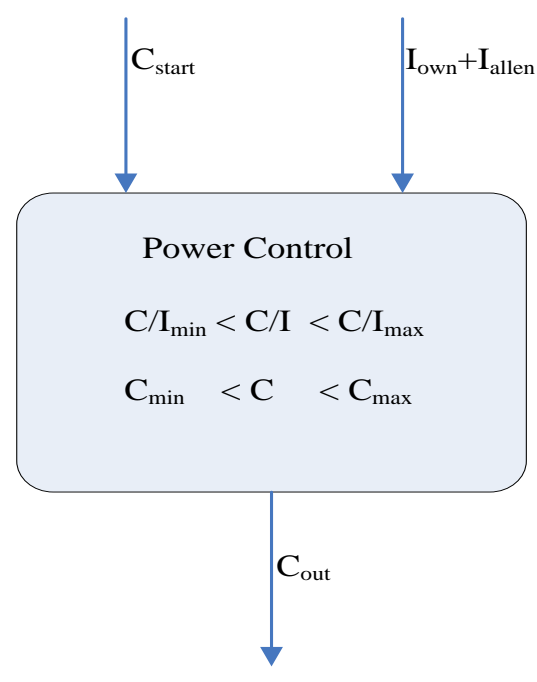

Figure 2. Power Control Mechanism

The model considers the interference caused by alien systems as well as the intrasystem interference. The control algorithm compares the $\mathrm{C} / \mathrm{I}$ value at the receiver with the minimum required and the maximum allowed $\mathrm{C} / \mathrm{I}$ value. In order to keep the received $\mathrm{C} / \mathrm{I}$ in its fixed boundaries the transmission power is controlled (if possible). Consequently the most important value during power control is the $\mathrm{C} / \mathrm{I}$. If the $\mathrm{C} / \mathrm{I}$ is in the required scope, the transmission power is varied to keep the received power in its fixed boundaries, too.

Figure 3 shows an example of the power algorithm. The axis of ordinate contains the $\mathrm{C} / \mathrm{I}$ threshold and the axis of abscissa contains the $\mathrm{C}$-thresholds. The two straight lines include all possible values for $\mathrm{C} / \mathrm{I}$ for a received interference power $\mathrm{I}_{1}$ and $\mathrm{I}_{2}$. The area defined by the thresholds is marked with grey. The control of the corresponding station's transmission power should be the point on the straight line across the marked area. Regarding the interference $I_{1}$, the transmission power must be pulled up until the 
minimum receiving power is reached. The upper $\mathrm{C} / \mathrm{I}$ threshold demand cannot be fulfilled here. Concerning $\mathrm{I}_{2}$, the grey marked area can be reached.

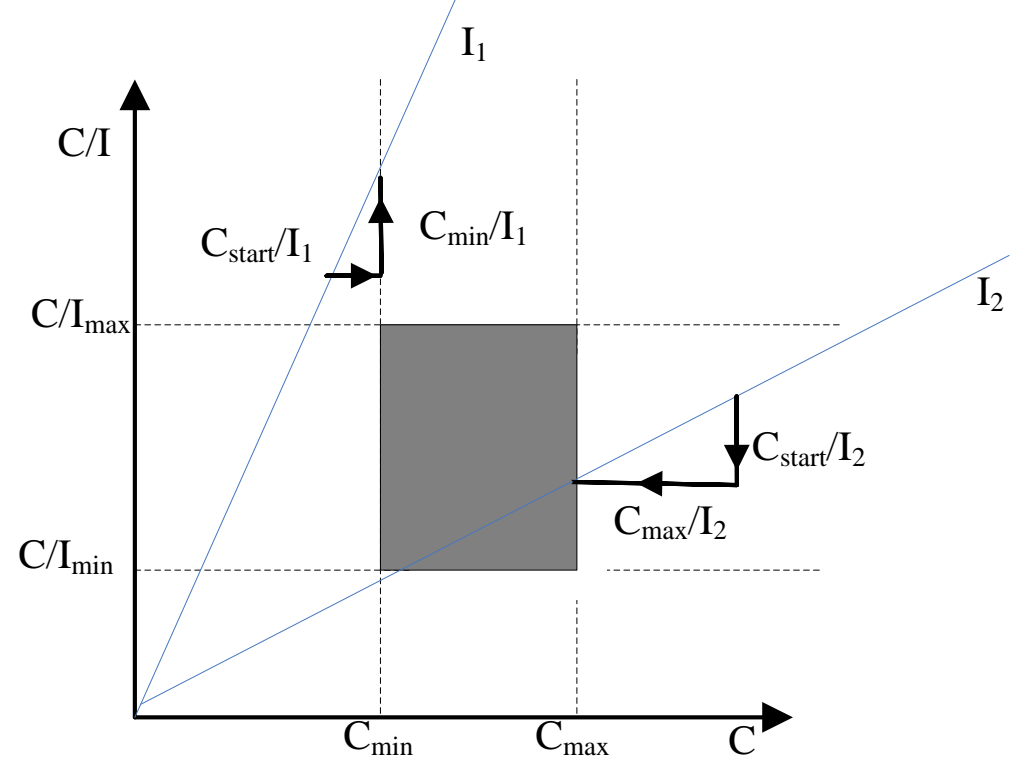

Figure 3. An Example of the Power Control

We will applied the following system parameters to analyze the experiment result.

\section{Table 1. System Parameters}

\begin{tabular}{|c|c|c|}
\hline Variable Signal & Parameter (Unit) & Value \\
\hline $\mathrm{R}$ & Femtocell Radius (m) & 30 \\
\hline f & FAP carrier frequency $(\mathrm{GHz})$ & 2 \\
\hline $\mathrm{P}_{\max }$ & Femtocell user transmission power(W) & 10 \\
\hline $\mathrm{M}$ & $\begin{array}{l}\text { pseudo random cycle number By Monte } \\
\text { Carlo method }\end{array}$ & 100 \\
\hline $\mathrm{K}$ & power iterative number & 100 \\
\hline $\mathrm{T}$ & FAP number in two-tier netwoks & 20 \\
\hline $\mathrm{N}$ & femtocell user number in a FAP area & 10 \\
\hline $\mathrm{F}(\mathrm{x})$ & $\begin{array}{l}\text { Whether femtocell user transmission signal } \\
\text { or not }\end{array}$ & Bernoulli distribution \\
\hline $\mathrm{n}$ & $\begin{array}{l}\text { Number of every femtocell user transmission } \\
\text { signal }\end{array}$ & 10 \\
\hline$q$ & Failure possibility of transmission signal & 0.3 \\
\hline $\mathrm{k}$ & $\begin{array}{c}\text { Failure Number of transimission signal of } \\
\text { every user in one FAP area }\end{array}$ & 4 \\
\hline$\Gamma_{\max }$ & Max SINR threshold & $\Gamma^{*}+2 \mathrm{~dB}$ \\
\hline$\Gamma_{\min }$ & Min SINR threshold & $\Gamma^{*}-2 \mathrm{~dB}$ \\
\hline$\beta_{i}$ & cost factor & 0.1 \\
\hline$\sigma^{2}$ & additive gaussian white noise power (W) & $1 \times 10^{-4}$ \\
\hline
\end{tabular}




\begin{tabular}{|l|c|c|}
\hline & FDD LTE simulation frequency $(\mathrm{GHz})$ & 2 \\
\hline & FDD LTE UL lowest frequency( MHz) & 1920 \\
\hline & FDD LTE UL highest frequency $(\mathrm{MHz})$ & 1980 \\
\hline & FDD LTE DL lowest frequency $(\mathrm{MHz})$ & 2110 \\
\hline & FDD LTE DL highest frequency( MHz) & 2170 \\
\hline & LTE bandwidth (MHz) & 15 \\
\hline & Number of RBs per LTE UE & 12 \\
\hline
\end{tabular}

Pareto optimality of the interference channel communication means that when two transmission powers are given, there is maximum value to overall channels achievable rate. It is only related to two inference channel parameters. When reaching the Pareto conditions, two channel capacity achieve a balance at the same time.

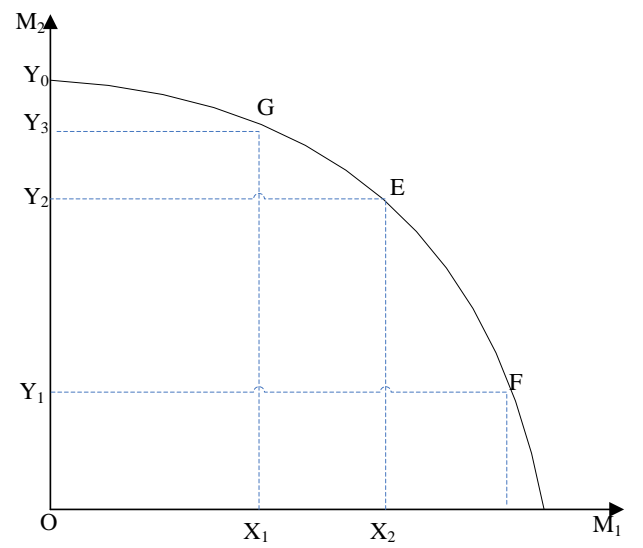

Figure 4. Max Achievable Rate and Pareto Point

In Figure 4, $M_{1}$-coordinate and $M_{2}$-coordinate show throughout of channel 1 and throughout of channel 2, respectively. At the point F, when throughout of channel 1 is preliminarily given, throughout of channel 2 can arrive the maximum $Y_{1}$ and vice versa. If one channel achievable rate increase, throughout of the other channel surely decrease. Slope of a curve shows the ratio of throughout from one channel to the other channel. It just shows the Pareto optimal thought.On the point E, the achievable rate of two channels equals and the sum of transmit power of two channels is minimum. So, the overall system is optimal at the moment. Eq.(28) use mathematical language to describe the physical meaning of point $\mathrm{E}$.

$M_{1}=M_{2}=\log _{2}\left(1+\sqrt{\frac{f h}{e g}}\right)$ 


\section{Conclusions}

We use the Pareto-optimal solution to analyze the achievable rate of the two-user interference channel. Our main contribution has derived a characterization of channel. When achievable rate of each channel equals, it is only related the channel parameters. The objective of this paper is to provide a theoretical method for simplifying more complicate technique that is used to mitigate interference and improve transmission rate. Our hope is that the results will be useful for future research.

\section{Acknowledgement}

This research has been supported by Research project of Tianjin science and technology development strategy (15ZLZLZF00310) and Soft project of Tianjin construction system(2015-soft 22).

\section{References}

[1] D. Tse and P. Viswanath, "Fundamentals of Wireless Communications", Cambridge Univ. Press, (2005).

[2] E. A. Jorswieck, E. G. Larsson and D. Danev, "Complete characterization of the pareto boundary for the MISO interference channel", IEEE Transactions on Signal Processing, vol. 56, no. 10, (2008), pp. $5292-5296$.

[3] K. Eleftherios and E. G. Larsson, "Efficient computation of the Pareto boundary for the MISO interference channel with perfect CSI", WiOpt 2010 - 8th Intl. Symposium on Modeling and Optimization in Mobile, Ad Hoc, and Wireless Networks, (2010), pp. 573-577.

[4] J. Lindblom, E. Karipidis and E. G. Larsson, "Closed-form parameterization of the Pareto boundary for the two-user MISO interference channel", ICASSP, IEEE International Conference on Acoustics, Speech and Signal Processing - Proceedings, (2011), pp. 3372-3375.

[5] K. Eleftherios, A. Gründinger, J. Lindblom and E. G. Larsson, "Pareto-optimal beamforming for the MISO interference channel with partial CSI", CAMSAP 2009 - 2009 3rd IEEE International Workshop on Computational Advances in Multi-Sensor Adaptive Processing, (2009),pp. 5-8.

[6] H. Boche and M. Schubert, "Complete characterization of the Pareto boundary of interference-coupled wireless systemswith power constraints- The log-convex case", ICASSP, IEEE International Conference on Acoustics, Speech and Signal Processing - Proceedings, (2009), pp. 3637-3640.

[7] T. C. Ji, H. Jonathan and N. Feng, "Wireless coexistence: Pareto optimality", GLOBECOM - IEEE Global Telecommunications Conference, vol. 6, (2004), pp. 3803-3807.

[8] D. P. Bertsekas, "Nonlinear Programming (Second ed.)", Cambridge, MA.: Athena Scientific., (1999).

[9] I. B. Vapnyarskii, "Lagrange multipliers", in Hazewinkel, Michiel, Encyclopaedia of Mathematics, Springer, (2001).

[10] H. Ozaki and Y. Omura.AN, "Advanced Nonlinear Signal Model To Analyze Pulsation-derived Photopletysmogram Signals", International Journal on Smart Sensing and Intelligent Systems, vol. 8, no. 2, (2015), pp. 921-943.

[11] S. Viswanath, M. Belcastro, J. Barton, B. O’Flynn, N. Holmes and P. Dixon, "Low-Power wireless Liquid Monitoring System Using Ultrasonic Sensors", International Journal on Smart Sensing and Intelligent Systems vol. 8, no.1, (2015), pp. 26-44.

[12] H.-C. Cheng and M.-C. Chiu, "A PC-based Wireless And Remote Comtrol For Shape Judgment Equipment Using A Zigbee Module", International Journal on Smart Sensing and Intelligent Systems vol. 7, no.3, (2014), pp. 1378-1400.

[13] T. Azuma, "A Method To Design Pid Controllers Using Frit-pso", International Journal on Smart Sensing and Intelligent Systems, vol. 8, no .4, (2015), pp. 1876-1895.

[14] M. Yang, "Optimal Cluster Head Number Based ON Entroy For Or Data Aggregation In Wireless Sensor Networks", International Journal on Smart Sensing and Intelligent Systems, vol. 8, no. 4, (2015), pp. 1935-1955.

[15] CF. Zhen, L. Wenyi and H. Wei, "The Implementation Of High Efficiency Wireless Sensor In Acoustic Object Tracking", International Journal on Smart Sensing and Intelligent Systems, vol. 8, no. 4, (2015), pp. 1997-2017. 